\title{
INFLUÊNCIA DOS HORMÔNIOS SEXUAIS NO CONSUMO DE OXIGÊNIO DE RATOS
}

\author{
INFLUENCE OF SEXUAL HORMONES ON OXYGEN CONSUMPTION IN RATS \\ INFLUENCIA DE LAS HORMONAS SEXUALES EN EL CONSUMO DE OXÍGENO EN RATAS
}

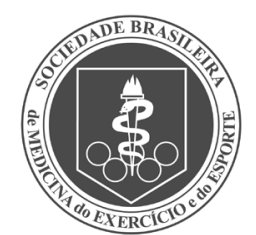

Artigo Original
Filipe Fernandes Conti (Educador Físico)

Iris Callado Sanches ${ }^{1}$ (Educadora Física)

Nathalia Bernardes ${ }^{1,2}$ (Educadora Física)

Guilherme Lemos Shimojo (Educador Físico)

Diego Mendrot Taboas Figueroa² (Educador Físico)

Rogério Brandão Wichi ${ }^{3}$

(Educador Físico)

Kátia De Angelis'

(Educadora Física)

\section{Laboratório de Fisiologia}

Translacional, Universidade Nove de Julho, São Paulo, SP, Brasil.

2. Laboratório de Hipertensão Experimental, Instituto do CoraçãoFMUSP, São Paulo, SP, Brasil.

3. Universidade Federal de Sergipe, São Cristóvão, SE, Brasil.

\section{Correspondência:}

Kátia De Angelis, Laboratório de Fisiologia Translacional, Universidade Nove de Julho (UNINOVE), Rua Vergueiro,235/249, Liberdade, 01504-001São Paulo, SP, Brasil. prof.kangelis@uninove.br.

\begin{abstract}
RESUMO
Introdução: Achados da literatura sugerem que oscilações dos hormônios sexuais femininos podem alterar o consumo máximo de oxigênio $\left(V_{2 \text { max }}\right)$. Objetivo: Comparar o $V_{2 \max }$ entre ratos machos $(M, n=8)$, fêmeas nas fases ovulatórias ( $\mathrm{OV}, \mathrm{n}=8$ ) ou não-ovulatórias ( $N O V, n=8$ ) do ciclo estral e ooforectomizadas (OS, n=8). Métodos: A avaliação do consumo de oxigênio foi realizada em esteira rolante com caixa metabólica acoplada. Resultados: Em repouso, - $\mathrm{VO}_{2}$ foi semelhante entre os grupos estudados. $\mathrm{O}$ grupo $\mathrm{M}$ apresentou maior $\mathrm{VO}_{2 \text { max }}$ em relação ao grupo NOV, mas valores semelhantes ao do grupo OV. O VO ${ }_{2 \max }$ foi menor no grupo ooforectomizado (OS: 62 $\pm 3 \mathrm{ml} . \mathrm{kg}^{-1}$. $\mathrm{min}^{-1}$ ) quando comparado aos demais grupos (M: 92 \pm 2 ; NOV: 77 \pm 4 ; OV: $87 \pm 3 \mathrm{ml} \cdot \mathrm{kg}^{-1} \cdot \mathrm{min}^{-1}$ ). Conclusões: Esses achados evidenciam que a variação hormonal decorrente das diferentes fases do ciclo estral e da privação dos hormônios ovarianos pode induzir alterações no consumo máximo de oxigênio em ratos.
\end{abstract}

Palavras-chave: consumo de oxigênio, ratos, diferenças sexuais, menopausa.

\section{ABSTRACT}

Introduction: Literature findings suggest that fluctuations of female sex hormones may change the maximum oxygen consumption $\left(\mathrm{VO}_{2 \text { max }}\right)$. Objective: To compare the $\mathrm{VO}_{2 \max }$ among male rats $(\mathrm{M}, n=8)$, female rats on ovulatory $(\mathrm{OV}, n=8)$ or non-ovulatory (NOV, $n=8$ ) phases of the estrous cycle and ovariectomized (OS, $n=8)$. Methods: The evaluation of oxygen consumption was performed on a treadmill coupled with metabolic box. Results: At rest $\mathrm{VO}_{2}$ was similar among groups. The $M$ group had a higher $\mathrm{VO}_{2 \max }$ compared to the NOV group, however showed similar values when compared to OV group. $\mathrm{Th} \mathrm{VO}_{2 \text { max }}$ was lower in the ovariectomized group (OS: $62 \pm 3 \mathrm{ml} \cdot \mathrm{kg}^{-1} . \mathrm{min}^{-1}$ ) compared to other groups (M: 92 2 ; NOV: $77 \pm$ 4; OV: $\left.87 \pm 3 \mathrm{ml}^{\mathrm{kg}} \mathrm{g}^{-1} . \mathrm{min}^{-1}\right)$. Conclusions: These findings suggest that hormonal variation resulting from different phases of the estrous cycle and ovarian hormone deprivation can induce changes in maximal oxygen uptake in rats.

Keywords: oxygen consumption, rats, sex differences, menopause.

\section{RESUMEN}

Introducción: Hallazgos de la literatura sugieren que oscilaciones de las hormonas sexuales femeninas pueden alterar el consumo máximo de oxígeno $\left(V_{2}{ }_{2 \max }\right)$. Objetivo: Comparar el $V_{2 \max }$ entre ratas machos $(M, n=8)$, hembras en las fases ovulatorias (OV, $n=8)$ o no ovulatorias (NOV, $n=8)$ del ciclo estral y ooforectomizadas (OS, n=8). Métodos: La evaluación del consumo de oxígeno fue realizada en cinta rodante con caja metabólica acoplada. Resultados: En reposo, el $\mathrm{VO}_{2}$ fue semejante entre los grupos estudiados. El grupo M presentó mayor $\mathrm{VO}_{2 \max }$ en relación al grupo NOV, pero valores semejantes a los del grupo OV. El VO ${ }_{2 \max }$ fue menor en el grupo ooforectomizado (OS: 62 \pm 3 ml. $\mathrm{kg}^{-1} \cdot \mathrm{min}^{-1}$ )

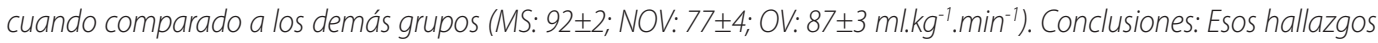
evidencian que la variación hormonal proveniente de las diferentes fases del ciclo estral y de la privación de las hormonas ováricas puede inducir alteraciones en el consumo máximo de oxígeno en ratas.

Palabras clave: consumo de oxígeno, ratas, diferencias sexuales, menopausia.

\section{INTRODUÇÃO}

O consumo máximo de oxigênio $\left(\mathrm{VO}_{2 \max }\right)$ é um indicador de capacidade aeróbica, cujo valor numérico se relaciona com o nível de eficiência de utilização da energia aeróbia. Seu valor absoluto depende da capacidade física de cada indivíduo, sendo um parâmetro fisiológico que expressa a quantidade de oxigênio que o organismo consome ou utiliza'. O $\mathrm{VO}_{2 \max }$ é o produto do fluxo sanguíneo sistêmico (débito cardíaco) e da extração sistêmica de oxigênio (diferença arteriovenosa de oxigênio), podendo ser expresso pela equação: $\mathrm{VO}_{2 \max }=$ frequência cardíaca máxima versus volume sistólico máximo versus diferença arteriovenosa de $\mathrm{O}_{2}$ máxima². Essa variável representa a integração que existe entre o sistema cardiovascular, respiratório e muscular frente à demanda energética durante o exercício ${ }^{3}$.

A medida de $\mathrm{VO}_{2 \max }$ é um excelente parâmetro para quantificar a capacidade cardiorrespiratória, bem como o nível de condicionamento físico do atleta e do indivíduo acometido por alguma doença ${ }^{4}$. Assim, - $\mathrm{VO}_{2 \max }$ além de ser um indicador de desempenho é também um indicador de fatores de risco.

Existe um consenso de que as oscilações hormonais devido às alterações, principalmente, nos níveis de estrogênio e progesterona, afetam a fisiologia feminina. Adicionalmente, estudos sugerem que o desempenho físico pode variar consideravelmente durante as distintas fases do ciclo menstrual, muito provavelmente em função das variações de temperatura corporal e de metabolismo que acompanham as curvas hormonais ${ }^{5-7}$. 
Dessa forma, o objetivo deste estudo foi comparar o consumo máximo de oxigênio entre ratos machos saúdaveis, fêmeas saudáveis nas fases ovulatórias ou não-ovulatórias do ciclo estral e ooforectomizadas.

\section{MATERIAIS E MÉTODOS}

Foram utilizados 32 ratos Wistar provenientes do biotério da Universidade São Judas Tadeu de São Paulo, SP, Brasil. Os animais foram mantidos agrupados em ambiente com temperatura controlada $\left(22-24^{\circ} \mathrm{C}\right.$ ) e com luz controlada em ciclo de 12 horas (claro/escuro). Água e comida foram oferecidas de modo irrestrito, sendo que a dieta foi normoprotêica. O presente projeto foi aprovado pelo Comitê de Ética em Pesquisa da Universidade São Judas Tadeu, com o parecer número 01/2008. Os animais foram divididos nos seguintes grupos experimentais ( $n=8$ em cada grupo): machos saudáveis (M), fêmeas saudáveis nas fases ovulatórias do ciclo estral (OV), fêmeas saudáveis nas fases não ovulatórias do ciclo estral (NOV), e fêmeas ooforectomizadas (OS). As avaliações do $\mathrm{VO}_{2}$ foram realizadas nos animais com 5 meses de vida.

A caracterização de cada fase do ciclo foi baseada na proporção de três tipos de células na secreção vaginal: epiteliais, corneificadas e leucócitos ${ }^{8}$. A secreção vaginal foi coletada com uma pipeta plástica com $10 \mu \mathrm{L}$ de solução salina introduzida superficialmente na vagina da rata. Essa gota de solução salina foi coletada e colocada em uma lâmina de vidro para a observação em um microscópio ótico (aumento de 400x). As avaliações foram realizadas nas fases ovulatórias (estro e proestro) e não ovulatória (diestro e metaestro) do ciclo estral.

\section{Ooforectomia}

Aproximadamente aos 90 dias de idade, após a certificação da vida reprodutiva, as ratas foram anestesiadas com cloridrato de cetamina (50mg/Kg Ketalar a 10\% ou 100mg/ml, Park-Davis) e cloridrato de xilazina (12 mg/Kg, Rompum a 2\% ou 20mg/ml, Bayer) e colocadas em decúbito dorsal para realização de uma pequena incisão $(1 \mathrm{~cm})$ em paralelo com a linha do corpo na pele e na musculatura no terço inferior na região abdominal. Os ovários foram localizados e foi realizada a ligadura dos ovidutos, incluindo os vasos sanguíneos. Os ovidutos foram seccionados e os ovários removidos. A musculatura e a pele foram suturadas e uma dose de antibiótico foi administrada (Benzetacil, $40000 \mathrm{U} / \mathrm{Kg}_{\text {, i.m) }}{ }^{9,10}$.

\section{Avaliação do consumo máximo de oxigênio $\left(\mathrm{VO}_{2 \text { máx }}\right)$}

Para realização do protocolo de avaliação do consumo máximo de oxigênio, os animais foram posicionados individualmente na caixa metabólica, sobre a esteira rolante, onde inicialmente foram coletados os valores de repouso. O tempo de observação foi de aproximadamente $30 \mathrm{~min}$. Após esse tempo, o teste de esforço foi imediatamente iniciado, e consistiu em submeter o animal à corrida na esteira com velocidade inicial de 0,3km/h por $3 \mathrm{~min}$, sendo esta carga incrementada em 0,3km/h a cada 3 min até que o animal atingisse a exaustão. A avaliação da capacidade física máxima foi feita por meio de respirometria aberta somente no SP1. A determinação metabólica de consumo de oxigênio foi de acordo com o método descrito por Brooks \& White ${ }^{11}$. O V $\mathrm{O}_{2}$ foi avaliado por meio de uma caixa metabólica conectada a um sensor de oxigênio (Power Lab, AD Instruments) que analisa continuamente as amostras das frações expiradas de oxigênio $\left(\mathrm{FeO}_{2}\right)$, bem como os valores das concentrações ambientais de oxigênio $\left(\mathrm{FiO}_{2}\right)$. Posteriormente, os valores de $\mathrm{VO}_{2}$ de cada animal foi calculado pela seguinte fórmula matemática: $\mathrm{VO}_{2}\left(\mathrm{mlO}_{2} \cdot \mathrm{kg}^{-1}\right.$. $\left.\min ^{-1}\right)=V E\left(\mathrm{FiO}_{2}-\mathrm{FeO}_{2}\right) / P C$, onde: VE: Fluxo da bomba de sucção (ml/ min); $\mathrm{FiO}_{2}$ : Fração inspirada de $\mathrm{O}_{2} ; \mathrm{FeO}_{2}$ : Fração expirada de $\mathrm{O}_{2} ; \mathrm{PC}$ : Peso corporal do animal (g). O VO $\mathrm{V}_{\text {reserva max }}$ foi obtido por meio da equação: $\mathrm{VO}_{2 \text { reserva max }}=\mathrm{VO}_{2 \max }-\mathrm{VO}_{2}$ de repouso.

\section{Análise estatística}

Os resultados são apresentados como média \pm erro padrão. O teste de análise de variância (ANOVA), seguido do post hoc de Student Newmann Keuls foi aplicado para análise dos dados. Valores de $p<0,05$ foram considerados significantes.

\section{RESULTADOS}

O peso corporal foi maior no grupo ooforectomizado quando comparado aos demais grupos (tabela 1).

Na figura 1 podemos observar o comportamento do $\mathrm{VO}_{2}$, durante o repouso e a cada incremento de carga do teste de esforço. Observa-se aumento do consumo de oxigênio em todos os grupos a cada aumento de carga durante o TE, observando tendência à estabilização de $\mathrm{VO}_{2}$ nas últimas cargas.

Como pode ser observado na tabela $1, \mathrm{OVO}_{2}$ de repouso foi similar entre os grupos. No entanto, o $\mathrm{VO}_{2 \max }$ e o $\mathrm{VO}_{2 \text { reserva max }}$ foram menores nas ratas na fase não ovulatória e nas ratas ooforectomizadas em relação ao grupo macho. Foi possível observar redução no $\mathrm{VO}_{2 \max }$ de 16,3\% no grupo não ovulatório e de 32,6\% no grupo ooforectomizado quando comparados ao grupo de macho. Além disso, os valores de $\mathrm{VO}_{2 \max }$ e o $\mathrm{VO}_{2 \text { reserva max }}$ também foram menores das ratas ooforectomizadas quando comparado às ratas nas fases ovulatória e não ovulatória do ciclo estral, apresentando redução significante no $\mathrm{VO}_{2 \max }$ de $19,4 \%$ quando comparado ao grupo não ovulatório e de 28,7\% quando comparado ao grupo ovulatório.

O grupo de machos atingiu $\mathrm{VO}_{2 \max }$ em uma maior velocidade quando comparado aos grupos de fêmeas (tabela 1).

Tabela 1. Peso corporal, consumo de oxigênio em repouso $\left(\mathrm{VO}_{2 \text { repouso }}\right)$, consumo máximo de oxigênio $\left(V O_{2 m a x}\right)$, consumo máximo de reserva de oxigênio $\left(V O_{2}\right.$ reserva max $=V O_{2 \max }$ $-\mathrm{VO}_{2 \text { repouso }}$ ) e velocidade na qual se atingiu o $\mathrm{VO}_{2 \text { max }}$ avaliado nos grupos $\mathrm{M}$ (machos, $\mathrm{n}=8$ ), NOV (não-ovulatório, n=8), OV (ovulatório, n=8) e OS (ooforectomizado, n=8).

\begin{tabular}{|c|c|c|c|c|}
\hline & M & NOV & OV & OS \\
\hline Peso corporal (g) & $242 \pm 6$ & $260 \pm 4$ & $255 \pm 7$ & $284 \pm 7^{*}+\#$ \\
\hline $\mathrm{VO}_{\text {2repouso }}(\mathrm{ml} / \mathrm{kg} / \mathrm{min})$ & $35 \pm 1$ & $33 \pm 2$ & $37 \pm 4$ & $34 \pm 2$ \\
\hline $\mathrm{VO}_{2 \max }(\mathrm{ml} / \mathrm{kg} / \mathrm{min})$ & $92 \pm 2$ & $77 \pm 4 *$ & $87 \pm 3$ & $62 \pm 3 *+\#$ \\
\hline $\mathrm{VO}_{\text {2reserva max }}(\mathrm{ml} / \mathrm{kg} / \mathrm{min})$ & $57 \pm 2$ & $44 \pm 4^{*}$ & $49 \pm 5$ & $28 \pm 4$ *十\# \\
\hline Velocidade do $\mathrm{VO}_{2 \max }(\mathrm{Km} / \mathrm{h})$ & $1,6 \pm 0,05$ & $0,9 \pm 0,1^{*}$ & $0,9 \pm 0,1^{*}$ & $1,1 \pm 0,1^{*}$ \\
\hline
\end{tabular}

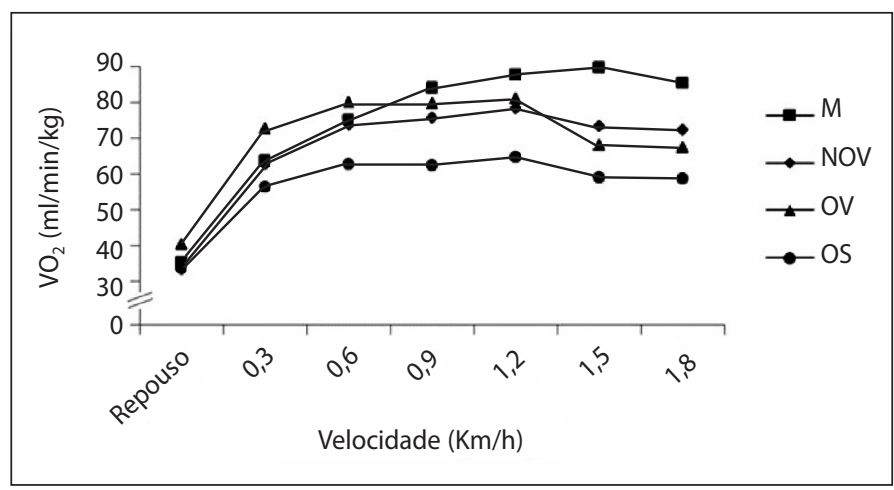

Figura 1. Consumo de oxigênio $\left(\mathrm{VO}_{2}\right)$ durante $\mathrm{o}$ teste de esforço realizado nos grupos de ratos machos $(M, n=8)$, ratas fêmeas nas fases não-ovulatórias (NOV, $n=8)$ e ovulatórias do ciclo estral (OV, $n=8)$ e ratas fêmeas ooforectomizadas $(O S, n=8)$.

\section{DISCUSSÃO}

O teste de esforço físico (TE) é um dos exames não invasivos mais utilizados para avaliar pacientes com doença cardiovascular. O TE tem por objetivo submeter o paciente a estresse físico, com finalidade de avaliar a resposta clínica, hemodinâmica, eletrocardiográfica e metabólica ao esforço. Essa avaliação permite detectar isquemia miocárdica, arritmias cardíacas, distúrbios hemodinâmicos esforço-induzidos, permitindo o diagnóstico e prognóstico das doenças cardiovasculares, bem como a prescrição de exercícios ${ }^{12}$. Vale lembrar que o consumo de oxigênio representa, hoje, não só um indicador de desempenho, mas um marcador prognóstico em cardiopatas ${ }^{3,13}$. Recentemente, demonstramos correlação positiva entre a velocidade atingida no teste de esforço e o consumo de oxigênio em ratos ${ }^{14}$. 
Como o $\mathrm{VO}_{2 \max }$ é frequentemente expressado em quilogramas do peso corporal ( $\mathrm{ml} / \mathrm{Kg} / \mathrm{min}$ ), mudanças no peso corporal podem afetar o $\mathrm{VO}_{2 \max }{ }^{15}$. No presente estudo, o peso corporal foi maior nas ratas ooforectomizadas em relação aos demais grupos, o que já era esperado, pois diversos estudos demonstram que ratas após a privação dos hormônios ovarianos apresentam aumento significante do peso corporal9,10.

Vale ressaltar que os valores obtidos $\mathrm{VO}_{2 \text { de repousor }} \mathrm{VO}_{2 \max } \mathrm{eVO}_{2 \text { dereservamax }}$ no presente estudo estão de acordo com dados encontrados na literatura para ratos adultos normais ${ }^{14,16}$.

É consenso que homens possuem maior $\mathrm{VO}_{2 \max }$ que mulheres $1,17,18$. Essa diferença é resultante de maiores concentrações de hemoglobina, além de um volume sanguíneo maior em homens, o que resulta em mais $\mathrm{O}_{2}$ carreado e ofertado aos tecidos ${ }^{19,20}$. No presente estudo, o grupo de ratos machos apresentou maior $\mathrm{VO}_{2 \max }$ quando comparado aos grupos ovulatório e ooforectomizado, corroborando com dados presentes na literatura, observados em homens e mulheres.

Lebrun ${ }^{21}$ publicou uma revisão da literatura na qual analisou os efeitos do ciclo menstrual no desempenho atlético. Nessa revisão, a maioria dos achados relatou uma melhora de desempenho na fase pós-menstrual. Burrows et al. ${ }^{22}$, em outra revisão, desta vez sobre treinamento para corrida de longa duração, sugeriram que o ciclo menstrual, bem como o uso de contraceptivos orais, não afetam o desempenho das atletas. Da mesma forma, Janse De Jonge ${ }^{15}$ também não encontrou diferenças durante as diferentes fases do ciclo menstrual para as medidas de lactato, concentração de hemoglobina e $V_{2 \max }$ Por outro lado, Charkoudian \& Johnson ${ }^{7}$ demonstraram que o desempenho físico pode variar consideravelmente durante as distintas fases do ciclo menstrual. Além disso, Lebrun et al. ${ }^{23}$ observaram diferença, tanto para o $\mathrm{VO}_{2 \text { max }}$ relativo, quanto para o $\mathrm{VO}_{2 \max }$ absoluto nas diferentes fases do ciclo, sendo que durante a fase lútea foi observado redução da capacidade aeróbica. No presente estudo, observamos um aumento de $11,5 \%$ no $\mathrm{V}_{2 \max }$ nas ratas durante as fases ovulatórias quando comparado às fases não-ovulatória, no entanto, sem diferença estatística. Vale lembrar que a fase não-ovulatória em ratas corresponde à fase lútea em mulheres, período em que os níveis de estrogênio e progesterona estão elevados ${ }^{14,24}$

Além das flutuações hormonais decorrentes das diferentes fases do ciclo, há um período na vida da mulher denominado climatério.

\section{REFERÊNCIAS}

1. Aránguiz H, Garcia V, Rojas S, Salas C, Martinez R, Mac Millan M. Estudio Descriptivo, Comparativo y Correlacional del Estado Nutricional y Condición Cardiorrespiratoria en Estudiantes Universitarios de Chile. Rev Chil Nutr 2010;37(1):70-8.

2. Powers SK, HowleyET. Fisiologia do exercício: Teoria e aplicação ao condicionamento e ao desporto. São Paulo: Manole, 2009

3. Myers J, Gullestad L, Vagelos R, Do D, Bellin D, Ross H, Fowler MB. Clinical hemodynamic and cardiopulmonary exercise test determinants of survival in patients referred for evaluation of heart failure. Ann Intern Med. 1998;129:286-93.

4. Lange B, Bruy T. Exercise spirometry in medical practice. Rev Med Liege 2001;56(4):228-32.

5. Sarwar R, Niclos BB, Rutherford OM. Changes in muscle strength, relaxation rate and fatigability during the human menstrual cycle. J Physiol. 1996;493(1):267-72.

6. Tenaglia SA, McLellan TM, Klentroum PP. Influence of menstrual cycle and oral contraceptive on tolerance to uncompensable heat stress. Eur J Appl Physiol.1999;80:78-83.

7. Charkoudian N, Johnson JM. Female reproductive hormones and thermoregulatory control of skin blood flow. Exerc Sport Sci. 2000;28:108-12

8. Marcondes FK, Bianchi FJ, Tanno AP. Determination of the estrous cycle phases of rats: some helpful considerations. Braz J Med Biol Res.2002;62(4a):609-14.

9. Irigoyen MC, Paulini J, Flores $\sqcup$, Flues $K$, Bertagnolli M, Moreira ED, et al. Exercise training improves baroreflex sensitivity associated with oxidative stress reduction in ovariectomized rats. Hypertension 2005;46:998-1003.

10. Flues K, Paulini J, Brito S, Sanches IC, Consolim-Colombo F, Irigoyen MC, De Angelis K. Exercise training associated with estrogen therapy induced cardiovascular benefits after ovarian hormones deprivation. Maturitas 2010;65(3):267-71

11. Brooks GA, White TP. Determination of metabolic and heart rate responses of rats to treadmill exercise. J Appl Physiol 1978;45(6):1009-15.

12. Negrão CE \& Barreto ACP. Cardiologia do exercício: Do atleta ao cardiopata, São Paulo: Manole, 2005.

13. Armstrong LE, Brubaker PH, Otto RM. ACSM's Guidelines for Exercise Testing and Prescription. In American College of Sports Medicine. $7^{\text {th }}$ Edition. Edited by Baltimore: Lippincott Williams \& Wilkins; 2005:66-99.

14. Rodrigues B, Figueroa DMT, Mostarda C, Heeren MV, Irigoyen MC, De Angelis K. Maximal exercise test is a useful method for physical capacity and oxygen consumption determination in streptozotocindiabetic rats. Cardiovasc Diabetol. 2007;6:38.

15. Janse De Jonge, XAK. Effects of the menstrual cycle on exercise performance. Sports Med 2003;33(11):833-51.
O climatério é caracterizado pela diminuição da atividade ovariana, a qual ocorre de forma lenta, com redução progressiva dos níveis circulantes de hormônios sexuais femininos ${ }^{25}$. É um período que continua após a menopausa e segue até a velhice ${ }^{26}$. Durante este período há mudanças na composição corporal da mulher, incluindo aumento dos adipócitos totais e redistribuição da gordura abdominal ${ }^{27,28}$, o que está associado ao aumento do risco de aterosclerose, aumentando, assim, o risco do desenvolvimento de doenças cardiovasculares ${ }^{29}$.

Nosso grupo vem estudando os efeitos da privação dos hormônios ovarianos em modelo experimental, bem como o papel do treinamento e da terapia estrogênica em parâmetros cardiovasculares e de estresse oxidativo ${ }^{9,10}$. No presente estudo, a privação dos hormônios ovarianos induziu redução do $\mathrm{VO}_{2 \max }$ em relação aos demais grupos estudados, sugerindo, como se observa em mulheres menopausadas, que a privação crônica dos hormônios ovarianos induza redução da capacidade aeróbica ${ }^{30}$. Por outro lado, em um modelo experimental de menopausa, Flues et al. ${ }^{10}$ não observaram diferença na capacidade de exercício, avaliado pelo teste de esforço máximo em esteira, entre ratas saudáveis e ooforectomizadas.

\section{CONCLUSÃO}

Em conjunto, os achados do presente estudo sugerem que as flutuações hormonais decorrentes das diferentes fases do ciclo estral e da privação dos hormônios ovarianos podem induzir mudanças no $\mathrm{VO}_{2 \text { max }}$. No entanto, estudos futuros devem estabelecer os mecanismos envolvidos em tais alterações do consumo máximo de oxigênio.

\section{AGRADECIMENTOS}

Os autores agradecem o apoio financeiro à Fundação de Amparo à Pesquisa do Estado de São Paulo (FAPESP: 2005/60829-2; 2007/57595-5; 2011/16441-0; 2012/20141-5), ao Conselho Nacional de Desenvolvimento Científico e Tecnológico (CNPq) e à Coordenação de Aperfeiçoamento de Pessoal de Nível Superior (CAPES-PVE: 88881.062178/2014-01).

Todos os autores declararam não haver qualquer potencial conflito de interesses referente a este artigo.

16. Wisløff U, Helgerud J, Johan Kemi O, Ellingsen.O Intensity-controlled treadmill running in rats: $\mathrm{VO}_{2 \max }$ and cardiac hypertrophy. Am J Physiol (Heart Circ Physiol) 2001;280:H1301-H10.

17. Azevedo PHSM, Oliveira JC, Simões HG, Baldissera V, Perez SEA. Cinética do consumo de oxigênio e tempo limite na $\mathrm{VO}_{2 \text { max }}$ : comparação entre homens e mulheres. Rev Bras Med Esporte 2010;16(4):278-81.

18. Billat V, Beillot J, Jan J, Rochcongar P, Carre F. Gender effect on the relationship of time limit at 100\% $\mathrm{VO}_{2 \max }$ with other bioenergetic characteristics. Med Sci Sports Exerc. 1996;28:1049-55.

19. Akalan C, Kravitz L, Robergs RA. VO ${ }_{2 m a x}$ :Essentials of the most widely used test in exercise physiology. Health Fit J. 2004;8(3):5-9.

20. Herdy AH, Uhlendorf D. Valores de referência para o teste cardiopulmonar para homens e mulheres sedentários e ativos. Arq Bras Cardiol. 2011;96(1):54-9.

21. Lebrun CM. Effect of the different phases of the menstrual cycle and oral contraceptives on athletic performance. Sports Med. 1993;16(6):400.

22. Burrows M, Bird S. The physiology of the highly trained female endurance runner. Sports Med. 2000; 30(4):281-300

23. Lebrun CM, MCKenzie DC, Prior JC, Taunton JE. Effects of menstrual cycle phase on athletic performance. Med Sci Sports Exerc. 1995;27(3):437-44.

24. Spornitz UM, Socin CD, David AA. Estrous stage determination in rats by means of scanning electron microscopic images of uterine surface epithelium. Anat Rec.1999;254:116-26.

25. Fuchs FD, Travi GM, Vaz MG, Nogueira MC, Silva MR, Nerene LL. Terapia de Reposição Hormonal pós-menopausa: Efeitos sobre os fatores de risco e incidência de doenças cardiovasculares. Rev Pesq Med1995;29(1):37-40

26. De Lorenzi DRS, Danelon C, Saciloto B, Padilha I Jr. Fatores indicadores da sintomatologia climatérica. Rev Bras Ginecolog Obstet. 2005;27(1):12-9.

27. Poehlman ET, Toth MJ, Gardner AW. Changes in energy balance and body composition at menopause: a controlled longitudinal study. Ann Intern Med. 1995;123:673-5.

28. Svendsen OL, Hassager C, Christiansen C. Age- and menopause associated variations in body composition and fat distribution in healthy women as measured by dual-energy $x$-ray absorptiometry. Metabolism. 1995;44:369-73.

29. Bjorntorp P."Portal" adipose tissue as a generator of risk factors for cardiovascular disease and diabetes. Arteriosclerosis. 1990;10:493- 6.

30. Lynch NA, Ryan AS, Berman DM, Sorkin JD, Nicklas BJ. Comparison of VO2max and disease risk factors between perimenopausal and postmenopausal women. Menopause. 2002 Nov-Dec:9(6):456-62 